\title{
EMG Based Clinical Evaluation of IndoKnee
}

\author{
Ashish Sahani, Anil Kumar Jain, Nilesh Satiram Gupta, Aalok Pandya, Priya Agarwal, Ganesh Ram \\ Jangir
}

\section{ABSTRACT}

IndoKnee is an unpowered knee assisting device designed and patented by Newndra Innovations. In this paper, we report a clinical study to characterize the reduction in load on the major muscles of the knee from the use of this device. Thirty healthy subjects without any history of knee problems underwent this trial. The protocol consists of three sets of events which are a) without IndoKnee (WOD), b) with IndoKnee at a minimum level of support (WDmin) and c) with IndoKnee at a maximum level of support (WDmax). Each set consists of five sitting and five standing activities. Surface electromyography was recorded from six major knee flexion/extension muscles, which are Bicep Femoris (BF), Semitendinosus (ST), Semimembranosus (SM), Rectus Femoris (RF), Vastus Medialis (VM) and Vastus Lateralis (VL). Paired t-tests were performed on our comparison between WOD vs WDmin and WOD vs WDmax to establish a significant difference in the EMG peak amplitudes during sitting and standing activities. The average reduction in all the six muscles for sitting activity is about $15 \%$ for both level of support and the standing activity, it is about $15 \%$ at a minimum level of support and $20 \%$ at a maximum level of support. In our investigation, IndoKnee did effectively reduce the required muscular effort for the knee flexion/extension. Thus, IndoKnee may prove to be an effective device in reducing knee pain, supporting osteoarthritis patients and faster rehabilitation from knee injuries.

Keywords: Clinical trial, EMG, Knee support device, Rehabilitation.

Published Online: September 26, 2020

ISSN: 2593-8339

DOI: $10.24018 /$ ejmed.2020.2.5.492

Ashish Sahani*

Indian Institute of Technology Ropar,

India.

(e-mail: ashish.sahani@trpr.ac.in)

Anil Kumar Jain

Santokba Durlabhji Memorial Hospital, India.

Nilesh Satiram Gupta

Indian Institute of Technology Ropar, India.

Aalok Pandya

JECRC University, India.

Priya Agarwal

Santokba Durlabhji Memorial Hospital, India.

Ganesh Ram Jangir

Newndra Innovations, India.

*Corresponding Author

\section{INTRODUCTION}

The overall prevalence of knee pain in the general population is approximately $25 \%$ of adults [1]-[4]. The incidences of knee pain increase steadily with age and a large part of this group have pain associated with disability. Some elderly experience difficulty in their daily life activities due to reduced endurance, strength, injury-resistance of the knee. A very common problem among elderly, athletes, labourers and farmers are highly debilitating pain that affects an individual. Osteoarthritis is the common type of arthritis in both developed and developing countries More than 30\% of the general population aged greater than 50 years suffer from osteoarthritis [5]-[7]. Hamstring strains or the weakness in the hamstring happens to about $12-16 \%$ [8]-[10] of the athletes, hamstring weakness reinjures the knee repeatedly with the reinjure rate of $22-34 \%$ [10], [11]. Knee braces are one of the solutions for knee pain, some wear them to prevent knee injuries or during rehabilitation.

In the current state, various knee braces are depending upon there functionality, and the mechanism is based on pretensioning the resilient means. A variety of support devices, including various exoskeleton-based devices and powered devices, are made to increase the user's strength, fatigue resistance or other factors. Knee Braces are made from combinations of metal, foam, elastic material and straps. They come in different sizes, designed and some are even battery powered. Categories of knee braces are as follows Rehabilitative braces, for stabilizing knee by allowing limited movement; Unloader braces are designed for people who have arthritis in their knee for shifting the weight to the stronger area and also for the aged people; functional braces provide support to knees that have been injured in the past, for the stabilization and control movement [12]. Past studies have shown that the unloader type of knee braces are effective in reducing knee pain, especially in elderly and patients of osteoarthritis [13]-[16]. In addition to this which have reported faster rehabilitation time to use of knee braces [17][20] and the comparative evaluation of different functional and unloading were studied to know the effectiveness of knee braces [14], [21].

Newndra Innovation Pvt. Ltd. has proposed and patented an affordable, efficient and unpowered knee assisting device called "IndoKnee" [22]. This Knee assisting device was developed to increase the individual's strength and fatigue resilience, particularly targeted at people with weak hamstrings, quadratus Femoris and osteoarthritis. IndoKnee can be used as a functional, unloader or rehabilitative type of knee brace and also provides deformity correction support. As a supporting means, it is useful for many applications including kneeling, walking, climbing, frequent up and down bending, or other situations where large pressures and stress are exerted on the knee and legs. 
Fig. 1 shows the CAD model of IndoKnee. It consists of the angular and radial adjustment mechanism in the rack and a resilient mean with one end fixed and other free. One end of the flexible element is fixed on a hinge and other passes through the single point to the resilient. This single point makes a flexible element to pull the upper element for deflection. This deflection stores energy in resilient mean and provide restoring torque for repositioning to initial position [22]. This single point mechanism enables the user to adjust the support efficiency for flexion and extension. Here in this study, we employed an IndoKnee with two levels of support (minimum and maximum).

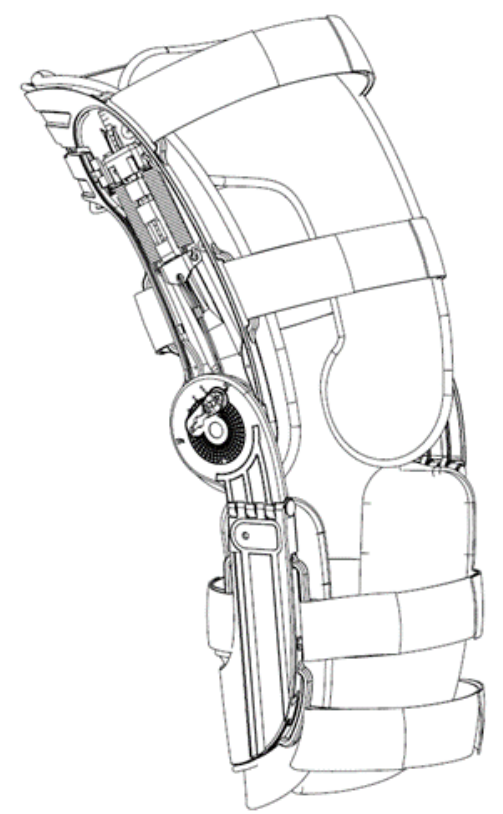

Fig. 1. IndoKnee: A device for Knee Support.

Electromyography (EMG) is a frequently employed method for characterizing the muscular force and fatigue [23], [24]. EMG amplitude measures the sum of action potentials of all the active motor units around the electrodes while muscle action is being performed.

To evaluate the effectiveness of IndoKnee in providing support to the major muscles around the knee, in this evaluation, we are using surface EMG signals for measuring the change in muscles tension during the flexion and extension of the knee.

\section{EXPERIMENTAL PROCEDURE}

\section{A. Subjects}

This study was conducted on 30 healthy participants (Summarized in Table I) with no history of major knee problems.

TABLE 1: SUMMARY OF SUBJECTS

\begin{tabular}{c|c}
\hline \hline No. of subjects & 30 \\
\hline Age & $28.84 \pm 7.5$ years \\
\hline Height & $167.03 \pm 6.7 \mathrm{~cm}$ \\
\hline Body mass & $61.38 \pm 7.4 \mathrm{Kg}$ \\
\hline $\begin{array}{c}\text { Height of waste from } \\
\text { ground level }\end{array}$ & $91.04 \pm 4.3 \mathrm{~cm}$ \\
\hline
\end{tabular}

\section{B. Instrumentation}

EMG data acquisition was done with iWorx ix-ta-220 Biopotential measurement system. The iWorx has two ports for 3-channel (2 electrodes each) preamplifiers called iWires. Both the iWires were used for acquiring six channels of EMG signals.

The EMG was measured from six major muscles of the knee which are Bicep Femoris (BF), Semitendinosus (ST), Semimembranosus (SM), Rectus Femoris (RF), Vastus Medialis (VM) and Vastus Lateralis (VL). The electrode placements are based on the SENIAM recommendation [25] and are illustrated in Fig. 2. Fourteen silver chloride $(\mathrm{AgCl})$ electrodes were used, two for input $(+\mathrm{V},-\mathrm{V})$ from each muscle and the remaining two for the reference or ground. Each ground is associated with one individual iWire device. The distance between two electrodes associated with each muscle fibre was approximately $4-5 \mathrm{~cm}$ to avoid the crosstalk from the neighboring muscle fibres. Goniometer available on the same iWorx platform was used to measure the bending angle of the knee time synchronized with the EMG data.

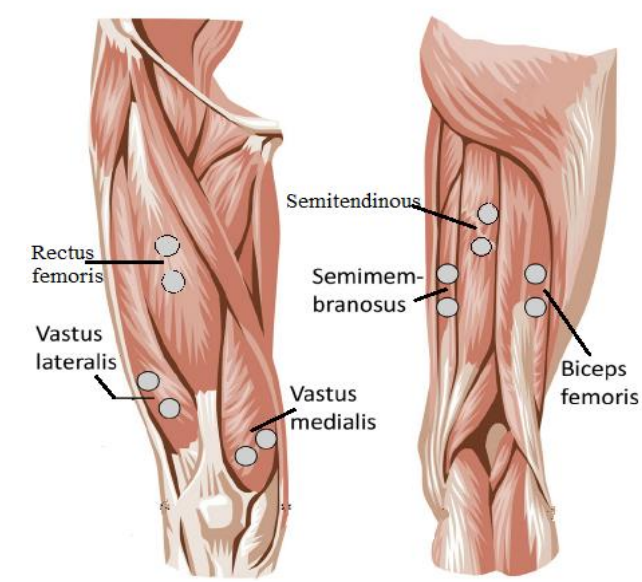

Fig. 2. Electrode placement location used for acquiring EMG signals.

We used LabScribe software for control and visualization of data acquisition through iWorx machine. The acquired data was exported in MATLAB readable '.mat' file format. Final processing and statistical analysis were done in MATLAB. The overall process flow of data acquisition and analysis is shown in Fig. 3.

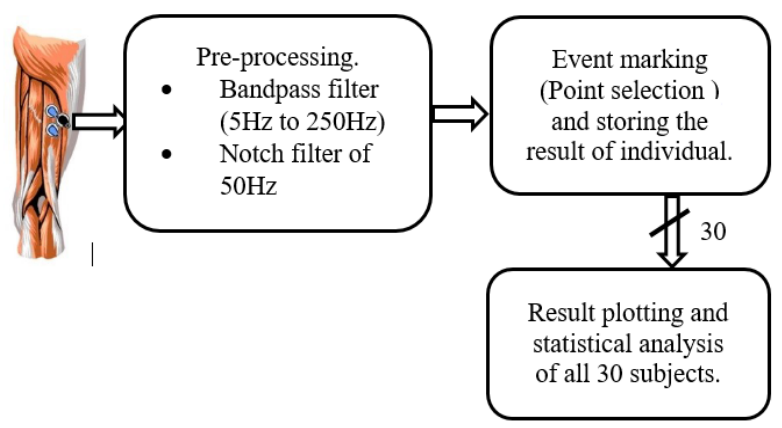

Fig. 3. Flowchart of designed experiment.

\section{Experimental design}

The protocol was approved by the IRB of SDMH, Jaipur. This study was registered with Central trial register of India (CTRI registration number CTRI/2019/07/020059 [Registered on 05/Jul/2019]). Before experimenting, subjects 
signed an informed consent form. The data of each subject's age, height, weight and lower limbs health history was recorded. The electrodes were placed at the designated locations (Fig. 2) after shaving and scrubbing the area for better conduction. After this, the subjects perform the sitting and standing activities while EMG and goniometer data is simultaneously acquired. The protocol has three sets of events; these are categorized as - without IndoKnee (WOD), with IndoKnee at minimum load support (WDmin) and with Indoknee at maximum load support (WDmax). Each set consists of five sitting down from standing position and five standing up from a seated position, with a resting time of 3 to 5 minutes between each set. The height of the seating stool from the ground level was $25 \mathrm{~cm}$. Picture of a subject undergoing the trial is shown in Fig. 4.

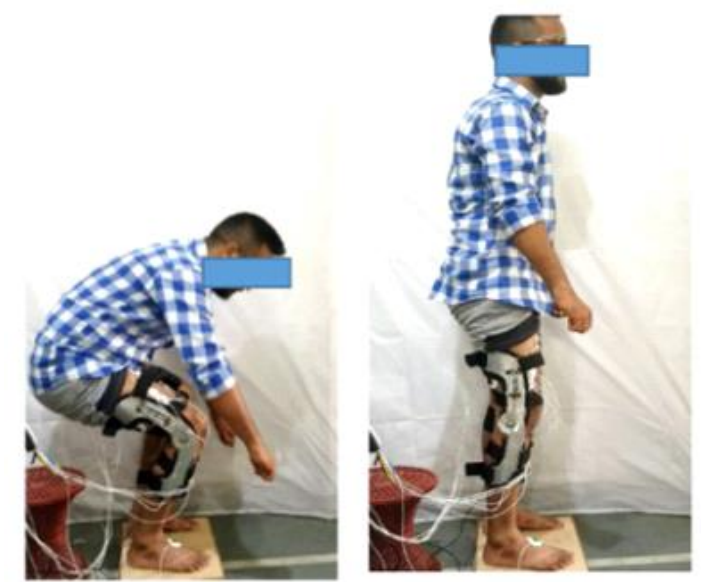

Fig. 4. An image of a subject performing designed experiment.

\section{DATA ANALYSIS}

\section{A. Pre-Processing}

The surface EMG signal was acquired from the iWorx system with the sampling rate of 1000 samples/sec [26] for all the muscles. Fig. 5 shows only a small section of raw EMG signal from all the six muscles. Full data is not shown due to readability reasons. This data was directly exported from iWorx into '.mat' format. Preprocessing of this raw signal was done to filter different types of noise such as power line artefacts and motion artefacts.

FIR hamming window bandpass filter was used with lower and upper cutoff frequencies like $10 \mathrm{~Hz}$ and $250 \mathrm{~Hz}$ respectively [26] and the $50 \mathrm{~Hz}$ notch filter is used remove the power line artefact and its odd harmonics $(150 \mathrm{~Hz}, 250 \mathrm{~Hz})$. The bandpass filter is used to remove the low-frequency and high-frequency artefacts from the signal. The lower cutoff frequency of the bandpass filter removes any DC offset and baseline drift associated with movement, preparation, etc. The higher cutoff frequency of bandpass minimizes the aliasing effect after the sampling of the signal. Fig. 6 shows the filtered EMG signal of the same section shown in Fig. 5. By observing both the raw and filtered data, we can determine that signal to noise ratio of the EMG signals are increased and the baseline noise is removed. Rectification of the EMG signal is one of the essential steps in EMG signal processing, in this step, all the negative part of the signal is made positive by taking the absolute value of all samples. Fig. 7 shows the rectified EMG signal.
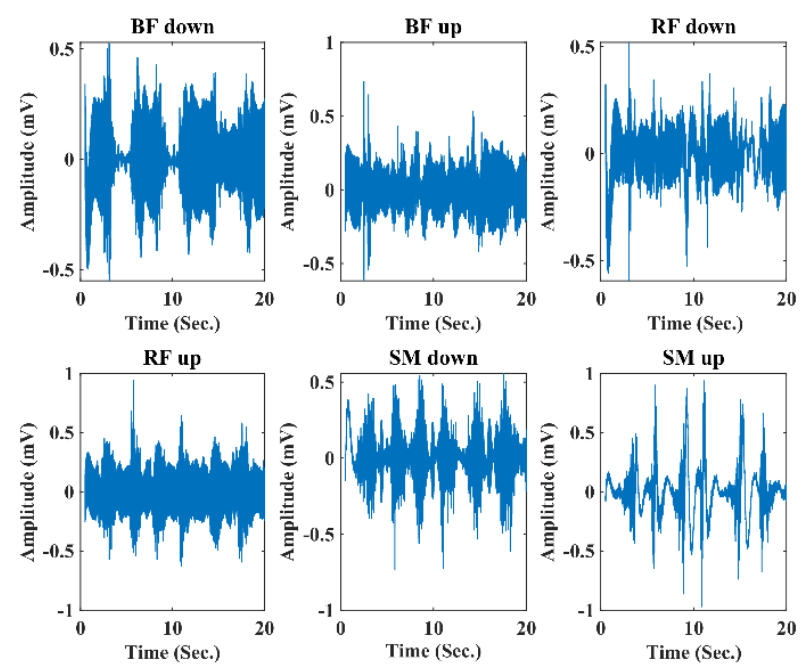

Fig. 5. Raw EMG signal.
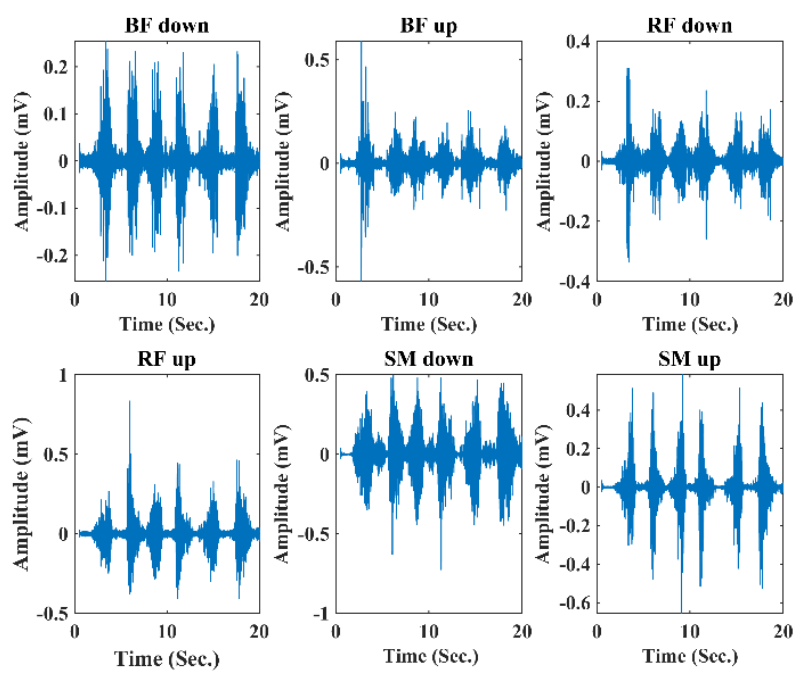

Fig. 6. Filtered EMG signal.
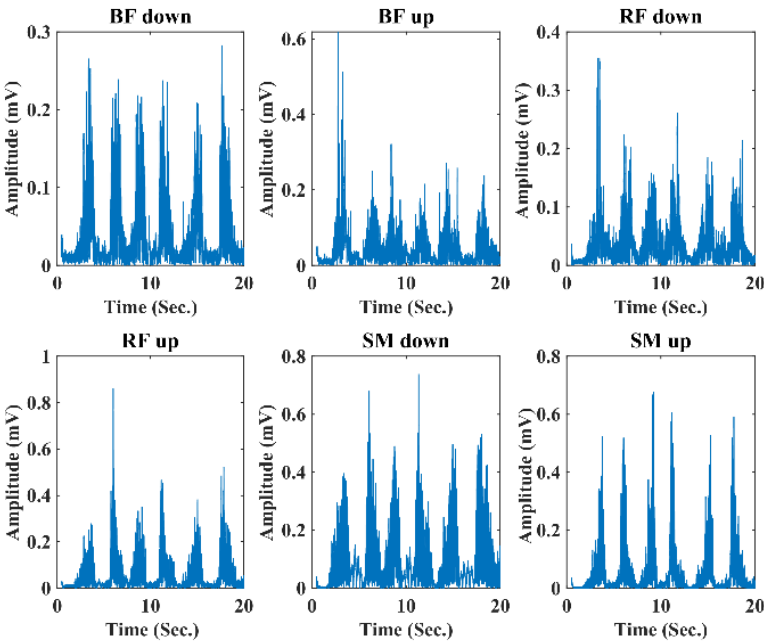

Fig. 7. Rectified EMG signal.

\section{B. Data Preparation}

We have overlaid EMG signals from all muscles over the goniometer signal in an interactive MATLAB plot an event marker was placed over the data during the change from 
standing to sitting or sitting to the standing position (Fig. 8). The section of EMG during sitting and standing for all the six muscle and all the iterations were stored in recognizable variables by the analyzing program. The signal in these variables is processed further to find the peak amplitude of EMG during each iteration of sitting and standing activity. For each muscle, we calculate the following six numbers:

1. Average of peak EMG for five iterations of sitting in WOD condition.

2. Average of peak EMG for five iterations of standing in WOD condition.

3. Average of peak EMG for five iterations of sitting in WDmin condition.

4. Average of peak EMG for five iterations of standing in WDmin condition.

5. Average of peak EMG for five iterations of sitting in WDmax condition.

6. Average of peak EMG for five iterations of standing WDmax condition.

As we are studying six muscles, we have a total of 36 observations per subject. These 36 measurements were then statistically analyzed, which is discussed in the following sections.

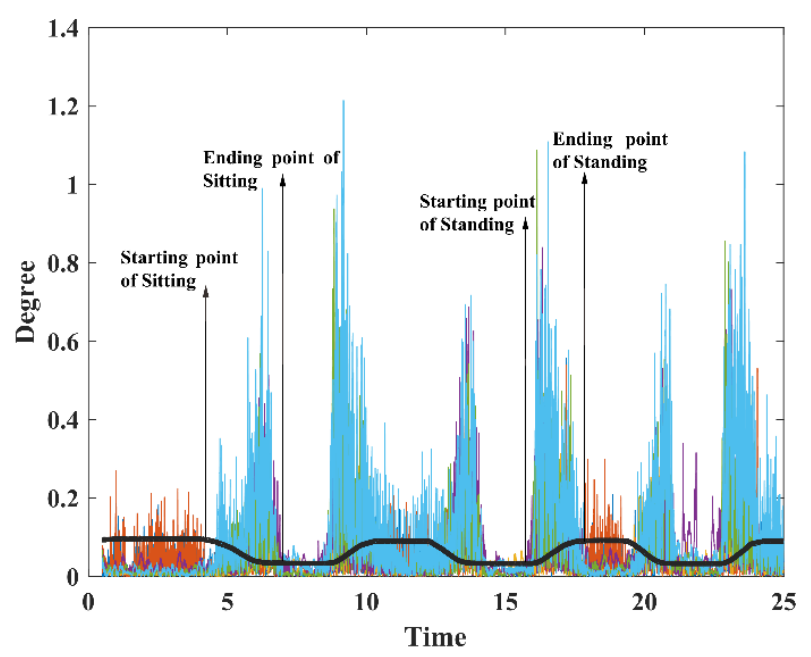

Fig. 8. Selection of point shows starting and ending point of each sitting down and standing up.

\section{RESULT}

Results are presented and briefly discussed in the following sections to make a clear comparison. Our results have box plots corresponding to six muscles which show the statistical information of clinical trials from all the thirty subjects (Fig. 9). The red line in between the boxes shows the median and black dot indicate the mean. We have also performed a paired t-test on our comparison between WOD vs WDmin and WOD vs WDmax to establish the significance of the reduction in EMG amplitude. Table 2 summarizes the percentage decrease and the corresponding p-values for all the muscles.

\section{A. Biceps Femoris}

The long head of the BF is a weaker knee flexor. When the hip is extended, both heads of the Biceps Femoris perform knee flexion. For sitting activity, when WOD is compared to WDmin, around 13\% decrease in EMG amplitude was observed and for WOD vs WDmax, we get a reduction of around $12 \%$. For standing activity, when WOD is compared to WDmin, around 8\% decrease in EMG amplitude was observed and for WOD vs WDmax, we get a reduction of around $12 \%$.

TABLE 2: AVERAGE PERCENTAGE DECREASE IN EMG VALUE AND ITS PARED T TEST P-VALUES

\begin{tabular}{|c|c|c|c|c|}
\hline & $\begin{array}{l}\text { WOD vs } \\
\text { WDMIN }\end{array}$ & P-Value* & $\begin{array}{l}\text { WOD vs } \\
\text { WDmax }\end{array}$ & P-Value* \\
\hline \multicolumn{5}{|c|}{ Sitting } \\
\hline$B F$ & 13.445 & 0.0171 & 11.579 & 0.0444 \\
\hline ST & 26.835 & $2.37 * 10^{-5}$ & 30.965 & $8.30 * 10^{-6}$ \\
\hline SM & 20.938 & 0.0076 & 18.387 & 0.00236 \\
\hline VL & 13.993 & 0.0023 & 19.181 & $1.38 * 10^{-4}$ \\
\hline $\mathrm{RF}$ & 25.849 & $3.20 * 10^{-5}$ & 34.042 & $2.06 * 10^{-5}$ \\
\hline VM & 18.997 & $1.03 * 10^{-7}$ & 26.795 & $4.90 * 10^{-9}$ \\
\hline \multicolumn{5}{|c|}{ Standing } \\
\hline$B F$ & 7.885 & 0.0114 & 11.529 & $3.34 * 10^{-4}$ \\
\hline ST & 17.085 & $5.46^{*} 10^{-4}$ & 24.654 & $1.49 * 10^{-4}$ \\
\hline SM & 12.449 & 0.0380 & 21.831 & 0.0068 \\
\hline VL & 10.048 & 0.0024 & 13.615 & 0.0011 \\
\hline $\mathrm{RF}$ & 18.620 & $2.02 * 10^{-4}$ & 23.635 & $3.56 * 10^{-4}$ \\
\hline VM & 14.072 & $7.08 * 10^{-5}$ & 22.59 & $8.34 * 10^{-8}$ \\
\hline
\end{tabular}

$*$ These are single tailed paired t-test because the EMG amplitude is always expected to go down with the device.

\section{B. Semitendinosus}

The ST muscle is one of three hamstring muscles. It lies between the BF and SM. These three muscles work collectively to flex the knee and extend the hip. In this muscle, the highest percentage of reduction was observed. For sitting activity, when WOD is compared to WDmin, around $27 \%$ decrease in EMG amplitude was observed and for WOD vs WDmax, we get a reduction of around $31 \%$. For standing activity, when WOD is compared to WDmin, around $17 \%$ decrease in EMG amplitude was observed and for WOD vs WDmax, we get a reduction of around $25 \%$.

\section{Semimembranosus}

The SM helps to straighten the hip joint and bend the knee joint. It also medially rotates the femur when the hip is extended. For sitting activity, when WOD is compared to WDmin, around $21 \%$ decrease in EMG amplitude was observed and for WOD vs WDmax, we get a reduction of around $19 \%$. For standing activity, when WOD is compared to WDmin, around $12.5 \%$ decrease in EMG amplitude was observed and for WOD vs WDmax, we get a reduction of around $21 \%$.

\section{Vastus Lateralis}

The quadriceps muscle group as a whole allows a person to stand up from sitting, walking and running. For sitting activity, when WOD is compared to WDmin, around $14 \%$ decrease in EMG amplitude was observed and for WOD vs WDmax, we get a reduction of around 19\%. For standing activity, when WOD is compared to WDmin, around $10 \%$ decrease in EMG amplitude was observed and for WOD vs WDmax, we get a reduction of around 14\%.

\section{E. Rectus Femoris}

The action of extending the knee from a seated position is primarily driven by VL, VM, and to a lesser degree by RF. For sitting activity, when WOD is compared to WDmin, around 26\% decrease in EMG amplitude was observed and for WOD vs WDmax, we get a reduction of around 34\%. For standing activity, when WOD is compared to WDmin, around $19 \%$ decrease in EMG amplitude was observed and for WOD vs WDmax, we get a reduction of around $24 \%$. 

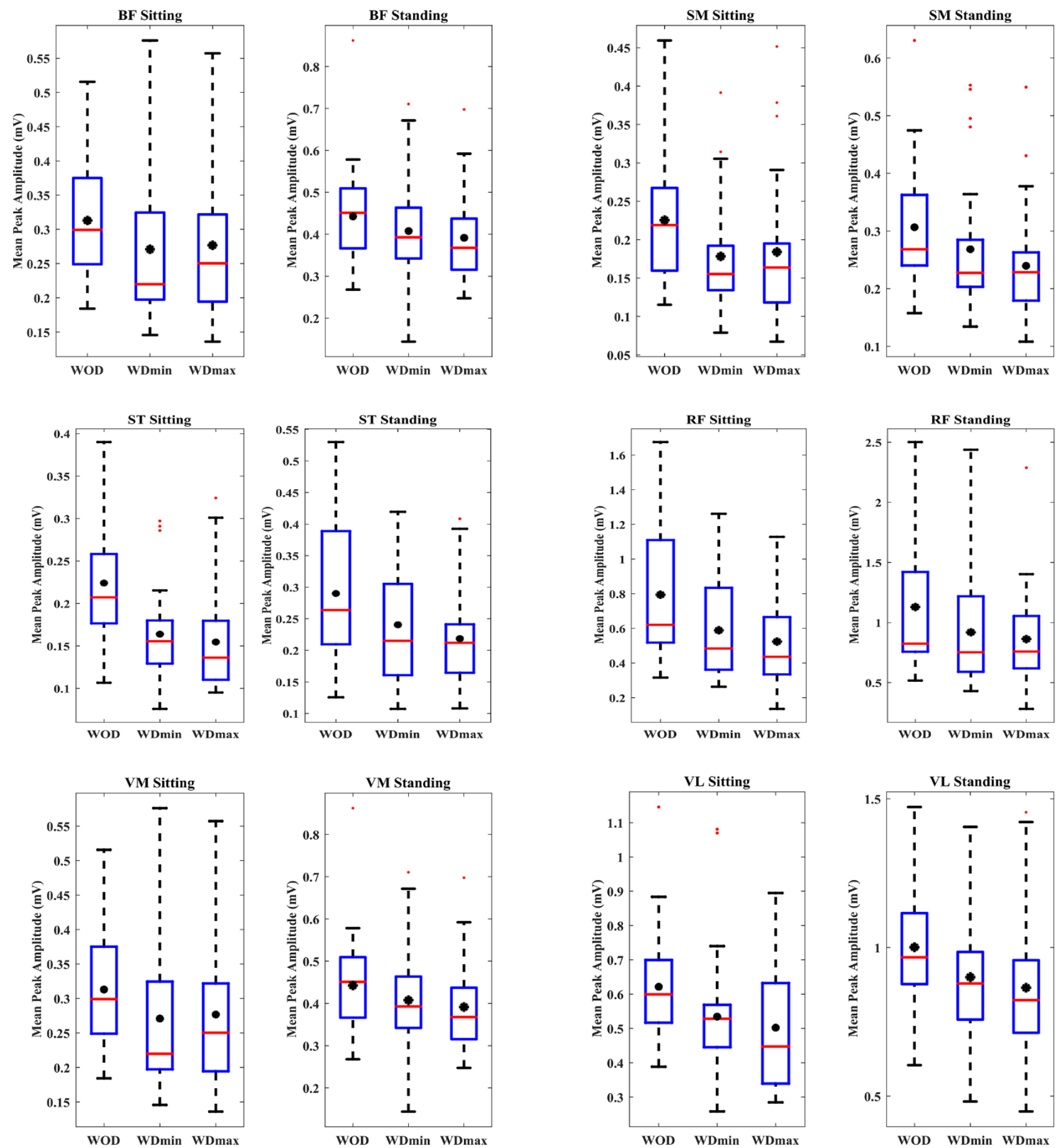

Fig. 9. Comparative box plots of reduction in EMG peak amplitude from all six muscles for all sets of sitting and standing activities.

\section{F. Vastus Medialis}

The VM is one of the four muscles in the anterior compartment of the thigh involved in knee extension along with the other muscles which make up the quadriceps muscle such as RF and VL. For sitting activity, when WOD is compared to WDmin, around $19 \%$ decrease in EMG amplitude was observed and for WOD vs WDmax, we get a reduction of around $27 \%$. For standing activity, when WOD is compared to WDmin, around $14 \%$ decrease in EMG amplitude was observed and for WOD vs WDmax, we get a reduction of around $23 \%$.

\section{DISCUSSION}

The investigation of EMG activities for six upper leg muscles was obtained in this study for evaluating the effect of IndoKnee on these muscles while using it. We have attempted to understand the muscle activity or change in the muscle force required for sitting down and standing up with IndoKnee at minimum support and at maximum support. As we know that the EMG amplitude is equivalent to the force applied by that muscle, thus, the percentage decrease shown in the results indicates a decrease in force for sitting and standing activity. We know that BF, ST and SM are mainly responsible for knee flexion, whereas RF, VM and VL are responsible for knee extension, thus the readings from the 
former three muscles are of particular interest while sitting and the later three during standing. Table 2 shows us the pvalues for the paired t-test done on the compared sets, we observed a statistically significant difference on the comparison $(p \leq 0.05)$, this shows that the difference we are getting are significant enough to declare that the device is reducing a load of all the six muscles during both sitting and standing activities.

For the sitting task, the support for BF, ST and SM muscles are more important as they have higher activity during the flexion of the knee. According to the result, the overall average reduction in EMG amplitude from these three muscles is about $20 \%$ during sitting exercise. During standing, RF, VL and VM muscles are more important as they have higher activity during knee extension. For these three muscles, the average reduction in EMG amplitude during standing activity is around $17 \%$. At the time of trials, we have asked our subject about their experience with the use of IndoKnee, some have said that they experienced high resistive force while sitting when the device is used at maximum efficiency (WDmax). For the sitting activity, BF and SM require more force (has more EMG amplitude values) during a sitting activity at WDmax as compared to WDmin. For the standing activity, all the muscles have shown positive response at both the support levels. Reduction in muscle force used for sitting activity is about $15 \%$ for both levels of support and for the standing activity, it's about $15 \%$ reduction at lower level of support and around $20 \%$ reduction at the higher level.

\section{CONCLUSION}

In this paper, we have investigated the efficacy of IndoKnee, an unpowered knee support device, designed by Newndra Innovation Pvt Ltd. For this, we conducted an EMG study of six major muscles of the knee during sitting and standing activities from thirty subjects without any prior history of knee problems. With the use of IndoKnee, we found a substantial reduction (of the order of 20\%) in muscle force requirements for sitting and standing activities. Thus we conclude, that IndoKnee can effectively reduce the load on the knee and potentially help in reducing knee pain, supporting osteoarthritis patients and faster rehabilitation from a knee injury

\section{REFERENCES}

[1] U. S. D. T. Nguyen, Y. Zhang, Y. Zhu, J. Niu, B. Zhang, and D. T. Felson, "Increasing prevalence of knee pain and symptomatic knee osteoarthritis: Survey and cohort data," Ann. Intern. Med., vol. 155, no. 11 , pp. 725-732, 2011.

[2] C. Jinks, K. Jordan, and P. Croft, "Measuring the population impact of knee pain and disability with the Western Ontario and McMaster Universities Osteoarthritis Index (WOMAC)," Pain, vol. 100, no. 12, pp. 55-64, 2002.

[3] L. R. Olson, S. E. S. Crawford, and J. L. Guth, "Changing issue agendas of women clergy,” J. Sci. Study Relig., vol. 39, no. 2, pp. 140 153, 2000.

[4] D. T. Felson and Y. Zhang, "An update on the epidemiology of knee and hip osteoarthritis with a view to prevention," Arthritis Rheum., vol. 41, no. 8, pp. 1343-1355, 1998.

[5] N. Yoshimura, S. Muraki, K. Nakamura, and S. Tanaka, "Epidemiology of the locomotive syndrome: The research on osteoarthritis/osteoporosis against disability study 2005-2015," Mod. Rheumatol., vol. 27, no. 1, pp. 1-7, 2017.

[6] M. Cross et al., "The global burden of hip and knee osteoarthritis: Estimates from the Global Burden of Disease 2010 study," Ann.
Rheum. Dis., vol. 73, no. 7, pp. 1323-1330, 2014.

[7] H. Ito et al., "Low back pain precedes the development of new knee pain in the elderly population; A novel predictive score from a longitudinal cohort study," Arthritis Res. Ther., vol. 21, no. 1, pp. 19, 2019.

[8] C. Woods, R. D. Hawkins, S. Maltby, M. Hulse, A. Thomas, and A. Hodson, "The Football Association Medical Research Programme: An audit of injuries in professional football - Analysis of hamstring injuries," Br. J. Sports Med., vol. 38, no. 1, pp. 36-41, 2004.

[9] J. H. M. Brooks, C. W. Fuller, S. P. T. Kemp, and D. B. Reddin, "Incidence, risk, and prevention of hamstring muscle injuries in professional rugby union," Am. J. Sports Med., vol. 34, no. 8, pp. 1297-1306, 2006.

[10] M. C. C. W. Elliott, B. Zarins, J. W. Powell, and C. D. Kenyon, "Hamstring muscle strains in professional football players: A 10-year review," Am. J. Sports Med., vol. 39, no. 4, pp. 843-850, 2011.

[11] N. Malliaropoulos, T. Isinkaye, K. Tsitas, and N. Maffulli, "Reinjury after acute posterior thigh muscle injuries in elite track and field athletes," Am. J. Sports Med., vol. 39, no. 2, pp. 304-310, 2011.

[12] familydoctor editorial staff, "Knee Bracing: What Works?," $\begin{array}{lll}\text { familydoctor.org, } & 2017 . & \text { [Online]. }\end{array}$ https://familydoctor.org/knee-bracing-what-works/.

[13] F. E. Pollo, J. C. Otis, S. I. Backus, R. F. Warren, and T. L. Wickiewicz, "Reduction of Medial Compartment Loads with Valgus Bracing of the Osteoarthritic Knee," Am. J. ofSports Med., vol. 30, no. 3, pp. 414-421, 2002.

[14] D. A. Dennis, R. D. Komistek, M. C. Nadaud, and M. Mahfouz, "Evaluation of Off-Loading Braces for Treatment of Unicompartmental Knee Arthrosis," J. Arthroplasty, vol. 21, no. 4 SUPPL., pp. 2-8, 2006.

[15] H. Matsuno, K. M. Kadowaki, and H. Tsuji, "Generation II knee bracing for severe medial compartment osteoarthritis of the knee," Arch. Phys. Med. Rehabil., vol. 78, no. 7, pp. 745-749, 1997.

[16] R. D. Komistek, "An In Vivo Analysis of the Effectiveness of the Osteoarthritic Knee Brace During Heel-Strike of Gait," J. Arthroplasty, vol. 14, no. 6, pp. 738-742, 1999.

[17] T. B. Birmingham, J. F. Kramer, and A. Kirkley, "Effect of a functional knee brace on knee flexion and extension strength after anterior cruciate ligament reconstruction," Arch. Phys. Med. Rehabil., vol. 83, no. 10, pp. 1472-1475, 2002.

[18] D. Van Tiggelen, E. Witvrouw, P. Roget, D. Cambier, L. Danneels, and R. Verdonk, "Effect of bracing on the prevention of anterior knee pain - A prospective randomized study," Knee Surgery, Sport. Traumatol. Arthrosc., vol. 12, no. 5, pp. 434-439, 2004.

[19] S. Brandsson, E. Faxén, J. file:///C:/Users/PM. L.9/Downloads/Tiggelen2004_Article_EffectOfBracingOnThePrevent on. pd. Kartus, B. I. Eriksson, and J. Karlsson, "Is a knee brace advantageous after anterior cruciate ligament surgery? A prospective, randomised study with a two-year follow-up," Scand. J. Med. Sci. Sport., vol. 11, no. 2, pp. 110-114, 2001.

[20] G. K. H. Wu, G. Y. F. Ng, and A. F. T. Mak, "Effects of knee bracing on the functional performance of patients with anterior cruciate ligament reconstruction," Arch. Phys. Med. Rehabil., vol. 82, no. 2, pp. 282-285, 2001.

[21] C. Beck, D. Drez, J. Young, W. D. Cannon, and M. L. Stone, "Instrumented testing of functional knee braces," Am. J. Sports Med., vol. 14, no. 4, pp. 253-256, 1986.

[22] G. R. Jangir, "A TORQUE ADJUSTING MECHANICAL ASSEMBLY IN APPARATUS FOR BODY SUPPORT, WO/2018/178897, 2017.

[23] A. L. Hof, "The relationship between electromyogram and muscle force," Sport. Sport., vol. 11, no. 03, pp. 79-86, 1997.

[24] G. Marco, B. Alberto, and T. M. Vieira, "Surface EMG and muscle fatigue: Multi-channel approaches to the study of myoelectric manifestations of muscle fatigue," Physiol. Meas., vol. 38, no. 5, pp. R27-R60, 2017.

[25] H. J. Hermens et al., "European Recommendations for Surface ElectroMyoGraphy," Roessingh Res. Dev., pp. 8-11, 1999.

[26] W. Rose, "Electromyogram analysis," Online course Mater. Univ. Delaware. Retrieved July, 5, 2016., 2011. 